\title{
New Strain of COVID-19 in India, Symptoms, Effects and Natural Therapeutics
}

\author{
Surinder Singh Bhinder* \\ Dr. S. S. Bhatnagar University Institute of Chemical Engineering and Technology, Panjab University, Chandigarh, India
}

*Corresponding author: Surinder Singh Bhinder, Dr. S. S. Bhatnagar University Institute of Chemical Engineering and

Technology, Panjab University, Chandigarh, India

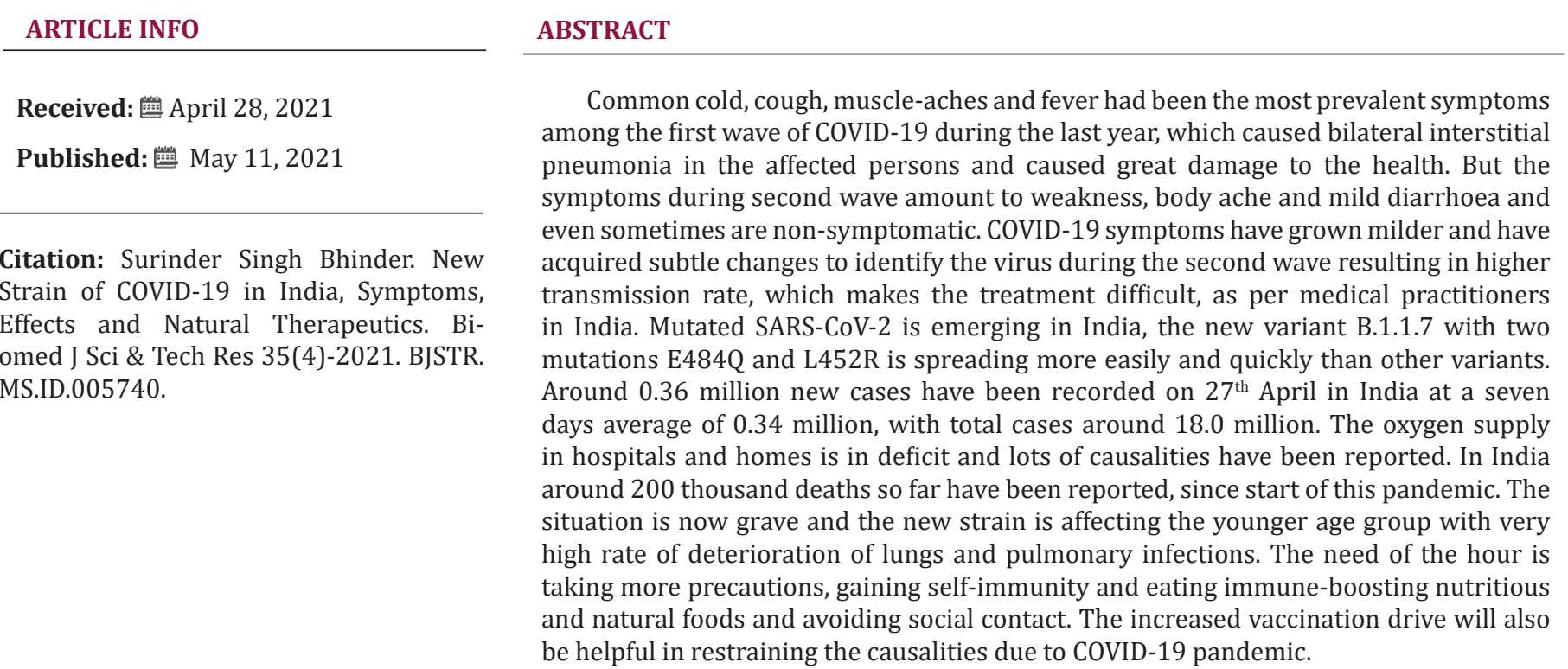

\section{Discussion}

COVID-19 cases (second wave) are on a surge around the globe and also in India having 1.3 billion population. The reason identified is the spread of double mutant strain B.1.1.7 along with earlier strains. The new strain affects the younger population in the age group of 18-45 years as compared to earlier strain which affected elderly i.e., more than 58-60 years of age and people with co-morbidities. Corona virus has caused great damage to health and at the same time economic resources [1]. The doctors have warned that the patients testing positive for B.1.1.7 strain will observe throat pain, weakness, mild cough, muscle aches and body fever than those who are infected by the earlier strain. In India people above the age of 45 have already been vaccinated and for younger people the vaccination drive is open from $1^{\text {st }}$ May 2021. A new COVID strain B.1.351 originated in South Africa also. This

strain possesses the capacity to cause Covid-19 in persons already recovered from coronavirus. Another important point about this strain is that it can potentially resist COVID-19 vaccines available till date. So, the upcoming lots of vaccines need to be upgraded to manage/control the detrimental effects of new strains of coronavirus disease in the persons infected. The use of steroidal drugs has also been utilized. The use of these steroidal drugs (anti-viral) like Remdesivir, Lectins etc. at appropriate time can save the lives of patients. It needs to be given to patients who are hospitalised, have a fall in oxygen saturation (below 85-90\%) and have infiltrates on the chest X-ray or CT-scan [2] as shown in Figure 1. If given early before the saturation level $\left(\mathrm{O}_{2}\right)$ falls, it has harmful effects. To prohibit COVID-19 from binding to the target cells, use of lectin along with glycans/mannose is recommended [3]. 


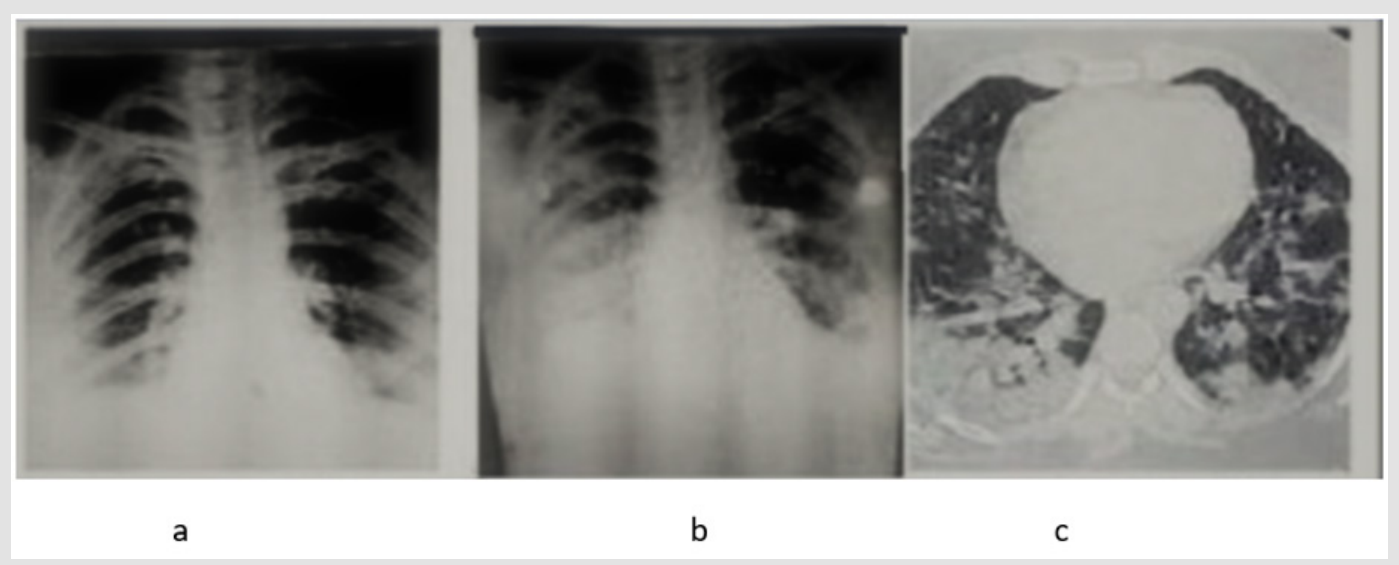

Figure 1:

a) X-ray on $9^{\text {th }}$ April 2021 showing normal lungs,

b) Second $X$-ray on $13^{\text {th }}$ April, 2021 showing big patches and

c) $\mathrm{CT}$ scan on $13^{\text {th }}$ April showing bilateral pneumonia with $80 \%$ Infection of a 32-year old female patient of Kota, India (Source Indian daily-Dainik Bhaskar). The infection might be due to the newly mutated strain, as such, fast deterioration of lungs in three days were rarely observed in the earlier COVID-19 strains.

\section{Long Term Effects}

It is observed that people get recovered from coronavirus disease in a fortnight or more, but the long-time effects are prevalent even after that. It has been reported that some people experience varied COVID-19 based effects for longer period of time after initial recovery like fatigue, daily tiredness, weakness, loss of stamina. These are identified as long term COVID-19 effects and cause damage to vital organs at the same time. Authentic research studies are being conducted further to investigate the long-term effects of COVID-19.

\section{Natural Therapeutics}

Use of natural sources (food materials) for immunity boosting and fruits and vegetable extracts have also been employed to contain corona virus. Plants based metabolites, e.g., withaferin, avicularin, guajaverin and asiatic acid possess maximum binding affinity with all key proteins of SARS-CoV-2 [4]. Identified compounds that can prohibit coronavirus in people are quercetin, isobavachalcone, tryptanthrin, myricetin, scutellarein, silvestrol, caffeic acid, psoralidin, saikosaponin $\mathrm{B}_{2}$ and griffithsin [5].

\section{Conclusion}

The new strain is causing severe illness in many of the patients, and it is sometimes difficult to detect. The optimum strategy can be the use of RT-PCR tests which can detect coronavirus in around 80 per cent of the cases, followed by clinical features and
CT scans/chest X-rays to rule out false negatives in symptomatic patients followed by a repeat test after 24 hours. Vaccination drive must be completed as early as possible in all age groups and ongoing precautions be continued. In this way accurate testing and vaccination can help preventing the rising surge of corona virus.

\section{Acknowledgement}

Panjab University, Chandigarh-for using digital and online resources.

\section{Conflicts of Interest}

There are no conflicts of interest.

\section{References}

1. Lauren L Patton (2021) Viral pandemics and oral health: Lessons learned from HIV to SARS-CoV-2. Oral Surgery, Oral Medicine, Oral Pathology and Oral Radiology 131(2): 149-153.

2. (2020) ACR recommendations for the use of chest radiography and CT for suspected Covid-19 infection.

3. Dawood Ghafoor, Sadia Ahmed, Nasib Zaman (2021) Lectins A Hope of Treatment for COVID-19. Am J Biomed Sci \& Res 12(3): 280-282.

4. Kazi Faizul Azim, Sheikh Rashel Ahmed, Anik Banika, Mostafigur Rahman Khan, Anamika Deba, et al. (2020) Screening and druggability analysis of some plant metabolites against SARS-CoV-2: An integrative computational approach. Inform Med Unlocked 20: 100367.

5. Janice S Mani, Joel B Johnson, Jason C Steel, Daniel A Broszczak, Paul M Neilsen, et al. (2020) Natural product-derived phytochemicals as potential agents against coronaviruses: A review. Virus Res 284: 197989. 


\section{ISSN: 2574-1241}

DOI: 10.26717/BJSTR.2021.35.005740

Surinder Singh Bhinder. Biomed J Sci \& Tech Res

(C) $(9)$ This work is licensed under Creative

Submission Link: https://biomedres.us/submit-manuscript.php

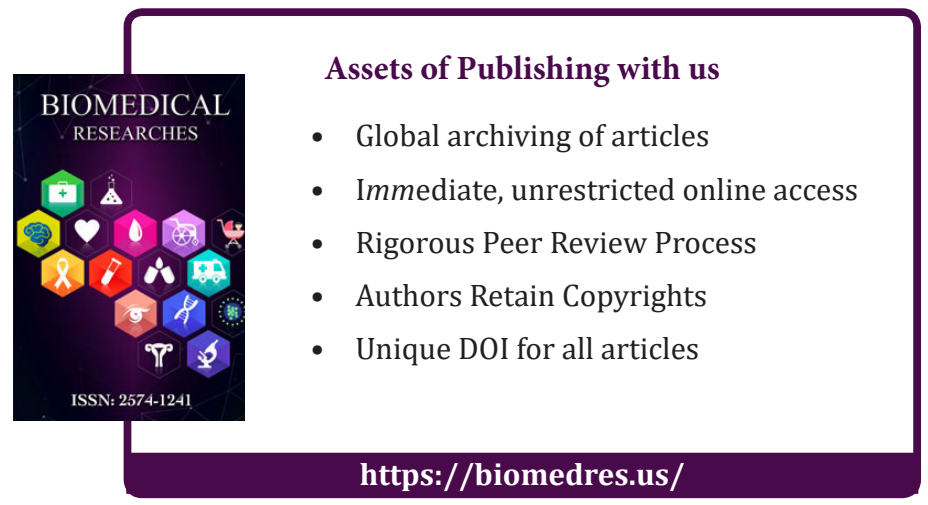

